\title{
Hybrid Control Accommodation for Water-asset Management of Hydraulic Systems Subjected to Large Operating Conditions
}

\author{
Eric Duviella, Pascale Chiron, Philippe Charbonnaud
}

\begin{abstract}
The Hybrid Control Accommodation (HCA) strategy was proposed to improve the water-asset management of hydraulic systems by resource allocation and setpoint assignment. Hydraulic system dynamics are taken into account during the setpoint assignment step which consists in controlling gates for large operating conditions. For hydraulic systems subjected to strong disturbances, transfer delays are variable, thus different operating modes must be considered. A multimodelling method, associated to a selection technique of transfer delay, allowing for the determination of the number of models, is proposed. The simulation results on the first reach of the Neste canal show the effectiveness of the HCA strategy.

Keywords: Hybrid control accommodation, resource allocation, setpoint assignment, multimodelling, water management.
\end{abstract}

\section{Introduction}

Hydrographic networks naturally convey water quantities upstream to downstream. They are equipped with dams, catchment areas, channels, etc., and are instrumented for water resource management and satisfaction of human activity needs. Several control methods have been designed [9], and are still designed $[8,2]$, to supply hydraulic systems with water quantities corresponding to the management objectives and rejecting disturbances. Other techniques, such as LPV regulation [10] or supervised internal multimodel controller [3] were recently proposed in order to consider these systems for different operating points. These control methods are accurate for a local control. However, they are not designed to allocate water quantities in excess towards the catchments areas, and water quantities in lack amongst the users. An original proposition for the management of such situations consists in recalculating setpoints according to the resource value (continuous dynamics) and to the resource state (discrete events) of the hydrographic systems. The supervision and hybrid control accommodation strategy by resource allocation and setpoint assignment proposed in [4] allows the water-asset management to take into account the management constraints. The strategy efficiency has been shown by simulation, in the case of an open-channel hydraulic system considering large operating conditions.

The problem addressed in this paper deals with the water-asset management of a hydraulic system subjected to scenarii with large disturbances. In the second section, the supervision and hybrid control accommodation strategy, well adapted to consider large operating conditions, is presented. In the third section, a multimodelling method is proposed for determining the number of Operating Modes (OM) necessary to represent open-surface hydraulic system dynamics, accurately. For each OM, the transfer delay identification is done in the fourth section. Finally, the effectiveness of this proposed strategy is shown by simulation on the first reach of the Neste canal.

\section{Hybrid control accommodation}

Hybrid Control Accommodation (HCA) strategy (see figure 1) allows the computation of new setpoints for each gate $G_{j}$ of hydraulic systems according to data measured on the measurement points $M_{i}$ (see figure 2). The setpoints are computed according to the resource state $e_{i}$, and taking into account management objectives $q_{j o b j}, \lambda_{j}$ and $\mu_{j}$ weekly fixed by the Management Objective Generation module. 


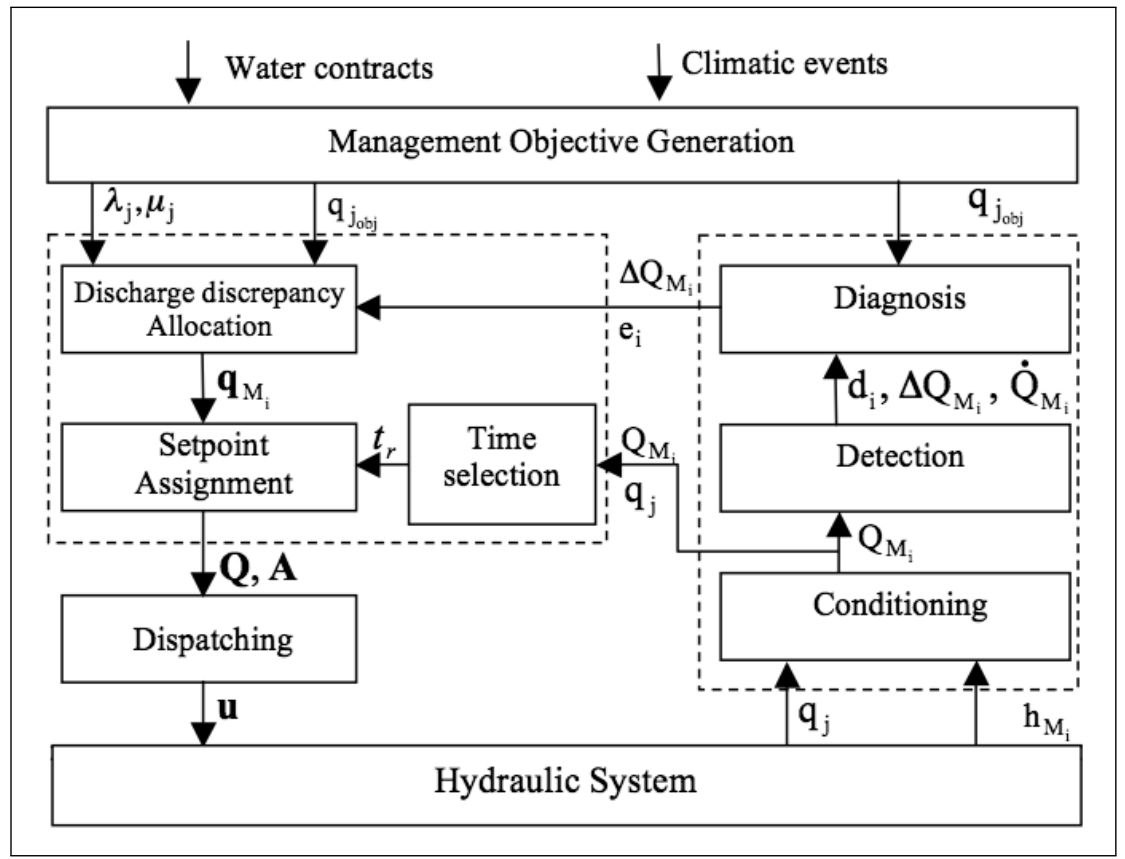

Figure 1: HCA strategy scheme.

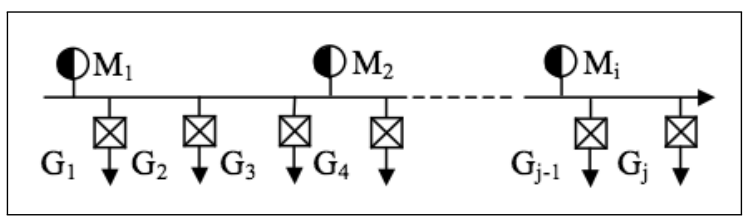

Figure 2: Open-surface hydraulic system equipped with measurement points $M_{i}$ and gates $G_{j}$. 
The resource state $e_{i}$ determination is carried out at the detection period $T_{e}$ by concurrent hybrid automata (see figure 3) designed for each measurement point $M_{i}$ [4]. The five pertinent states retained correspond respectively to no-discrepancy state $e_{0}$, two states where the discharge discrepancy is either positive $(e+)$ or negative $(e-)$ and constant $c$, and two states where the discharge discrepancy is either positive $(e+)$ or negative $(e-)$ and no constant $\neg c$. Transitions between states are defined as conditions on the measured discharge value and variation:

$$
\left\{\begin{array}{l}
d_{i}:\left[\left|\Delta Q_{M_{i}}\right|>t h_{i}\right], \\
\psi_{i}:\left[\Delta Q_{M_{i}}<0\right], \\
\omega_{i}:\left[\left|\dot{Q}_{M_{i}}\right|<d t h_{i}\right],
\end{array}\right.
$$

with $\Delta Q_{M_{i}}=Q_{M_{i}}-\sum_{j=n_{i}}^{n} q_{j o b j}$, where $Q_{M_{i}}$ is the measured discharge, $q_{j o b j}$ is the management objective of the gate $j, n_{i}$ the index of the first gate downstream the $i^{t h}$ measurement point $M_{i}, n$ the number of gates, $\dot{Q}_{M_{i}}$ the estimate derivative of $Q_{M_{i}}, t h_{i}$ and $d t h_{i}$ respectively the detection and diagnosis thresholds.

The discharge discrepancy allocation is carried out according to the resource state. When the state is $e_{0}$, the gate setpoints correspond to their discharge objective $q_{j o b j}$. When the state is $e \pm \wedge c$, the discharge discrepancy $\Delta Q_{M_{i}}$ is allocated amongst gates, according to their weights $\lambda_{j}$ and $\mu_{j}$, by optimizing, for each measurement point, a cost function. Finally, when the state is $e \pm \wedge \neg c$, the discharge discrepancy is allocated on gate each one on its turn [4]. At each detection date $k T_{e}$, the discharge discrepancy allocation leads to the allocation vector $\mathbf{q}_{M_{i}}^{k}$ :

$$
\mathbf{q}_{M_{i}}^{k}=\left[\begin{array}{llllllll}
0 & 0 & \ldots & 0 & q_{n_{i}}^{k} & q_{n_{i}+1}^{k} & \ldots & q_{n}^{k}
\end{array}\right]^{T},
$$

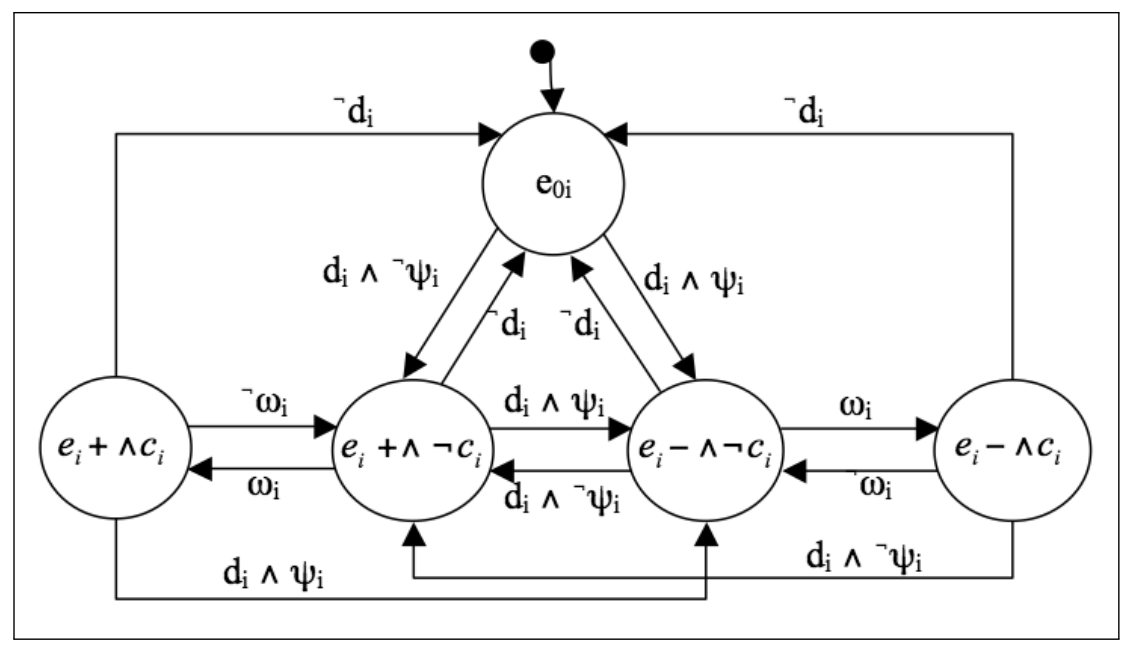

Figure 3: Hybrid automata for the measurement point $M_{i}$.

Then, to synchronize the control of the gate with the appearance of lacks or excess of the water due to the disturbances, the setpoints must be assigned at a date taking into account the transfer delays $T_{M_{i}, j}$ between the measurement point $M_{i}$ and the gate $G_{j}$. It is computed according to the relation (3):

$$
T_{M_{i}, j}=T_{M_{i}, n_{i}}+\sum_{r=n_{i}+1}^{j} t_{r}
$$

where $t_{r}$ is the time necessary for the water quantity to go from gate $G_{r-1}$ to gate $G_{r}$ (see Figure 4).

The delay $t_{r}$ depends on the physical characteristics of the hydraulic system and on the discharge value $Q_{r}$. Thereafter, the allocation vector $\mathbf{q}_{M_{i}}^{k}$ is associated with an allocation dates vector $\mathbf{T}$ arising 


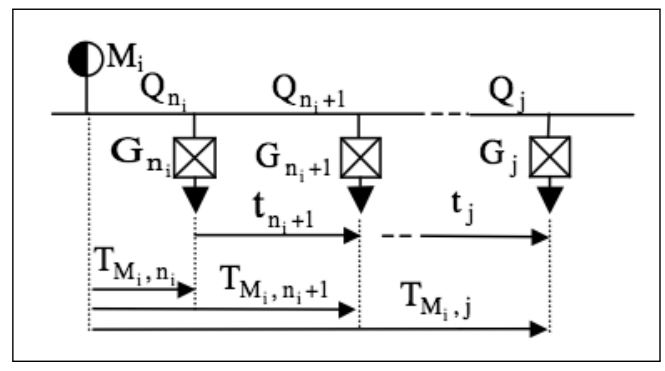

Figure 4: Time delays between $M_{i}$ and $G_{j}$.

from the transfer delay values $T_{M_{i}, j}$. Finally, taking into account the allocation date, the setpoints are sent to gates periodically. The control period $T_{c}$ is chosen as a multiple integer of $T_{e}$. In the next section, a multimodelling method is proposed to identify the system dynamics for several OM.

\section{Multimodelling steps}

The modelling of the free-surface hydraulic system dynamics is generally carried out starting from the diffusive wave equation (4) which is obtained by simplifying Saint Venant equations [1].

$$
\frac{d Q}{d t}+C \frac{d Q}{d x}-D \frac{d^{2} Q}{d x^{2}}=0
$$

where $C$ and $D$ are respectively the celerity and diffusion coefficients.

The diffusive wave equation can be linearized according to an operating discharge $Q_{e}$ [7], and the identified celerity and diffusion parameters, denoted $C_{e}$ and $D_{e}$, are expressed as:

$$
\left\{\begin{array}{l}
C_{e}=\frac{1}{L^{2} \frac{\partial J}{\partial Q_{e}}}\left[\frac{\partial L}{\partial x}-\frac{\partial J L}{\partial z}\right], \\
D_{e}=\frac{1}{L \frac{\partial J}{\partial Q_{e}}},
\end{array}\right.
$$

where $L$ is the surface width, $z$ is the discharge depth, $J$ is the friction slope expressed with the Manning-Strickler relation as $J=\frac{Q^{2} P^{\frac{4}{3}}}{K^{2} S^{\frac{10}{3}}}$ where $K$ is the Strickler coefficient, $P$ is the wetted perimeter and $S$ the wetted surface. The open-channel systems dynamics can be modelled by transfer functions, as:

$$
F(s)=\frac{e^{-\tau s}}{1+w_{1} s+w_{2} s^{2}}
$$

where the coefficients $w_{1}, w_{2}$ and the pure delay $\tau$ are computed according to the coefficients $C_{e}, D_{e}$, to the open-channel system length $X$, and to the adimensional coefficient $C_{L}=\frac{2 C_{e} X}{9 D_{e}}$; if $C_{L} \leq \frac{4}{9}, w_{2}=0$ and $\tau=0$, if $\frac{4}{9}<C_{L} \leq 1, w_{2}=0$.

This modelling method allows the identification of the free-surface hydraulic system dynamics with good accuracy only around an operating point. However, setpoint assignment must be done for hydraulic system subjected to large operating conditions. Thus, the hydraulic system dynamics must be identified for different OM. Based on the previous modelling method, the multimodelling approach consists in determining the number of $\mathrm{OM}$ and their corresponding operating points.

The celerity coefficient $C$ is a very relevant parameter of the hydraulic system dynamics. The model identified for each $\mathrm{OM}$ is available as soon as the error on the celerity coefficient is less than a predefined percentage $\Pi_{C}$. Thus, a validity domain is defined for each OM, and the number of OM which are necessary to identify the dynamics with a good accuracy, is determined. To limit the switching between two OM, an interval $\Delta_{C}$ is shared by two successive OM validity domains. The selection of parameters $\Pi_{C}$ and $\Delta_{C}$ is carried out taking into account the system dynamics. The multimodelling steps are described 
Table 1: Multimodelling algorithm.

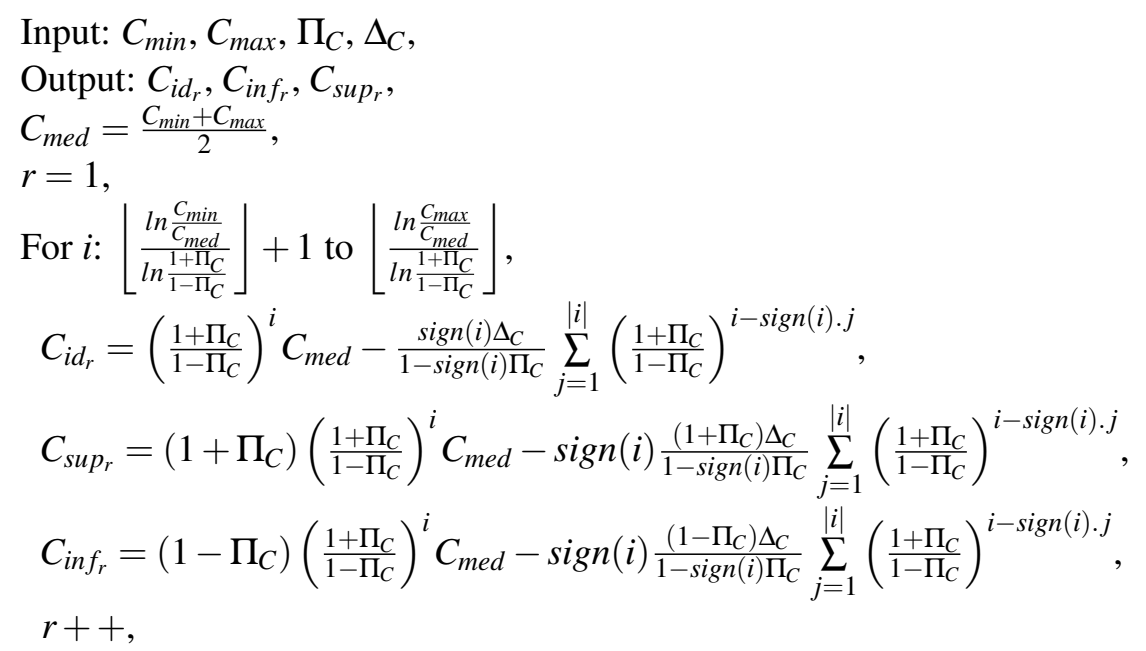

EndFor

by an algorithm (see Table 1), where the OM are determined starting with $C_{m e d}$. This one is computed with the parameters $C_{\min }$ and $C_{\max }$ corresponding respectively to the minimum and maximum discharges of the system. The algorithm requires the definition of $\lfloor x\rfloor$ which corresponds to the integer part of $x$, and $\operatorname{sign}(x) \operatorname{such}$ as $\operatorname{sign}(x)=\frac{|x|}{x}$.

Then, according to $C_{i d_{r}}$, the water elevation $z_{i d_{r}}$ of each $r^{\text {th }} \mathrm{OM}$ is determined, with one millimeter accuracy, using the digital resolution of the relation (7) with the Newton method.

$$
C_{i d}=\frac{\sqrt{J} K S^{\frac{5}{3}}}{P^{\frac{2}{3}} L^{2}}\left[-\frac{1}{2} \frac{\partial L}{\partial z}-\frac{L}{3 P}\left(2 \frac{\partial P}{\partial z}-5 \frac{P}{S} \frac{\partial S}{\partial z}\right)\right] .
$$

Then, the water elevation $z_{i d_{r}}$ value is used to compute the discharge $Q_{i d_{r}}$ with the relation (8). The same steps are used for parameters $C_{\text {inf }}$ and $C_{\text {sup }}$ to obtain the domain validity boundaries of the OM: $\left[Q_{\text {inf }_{r}} ; Q_{\text {sup }_{r}}[\right.$.

$$
Q_{i d_{r}}=\frac{\sqrt{J} K S^{\frac{5}{3}}}{P^{\frac{2}{3}}} .
$$

Finally, the celerity and diffusion coefficients $C_{i d_{r}}$ and $D_{i d_{r}}$ (5), and the transfer function parameters $w_{1}, w_{2}$ and $\tau(6)$ are computed according to the discharge $Q_{i d_{r}}$. These parameters allow for the computation and the selection of the transfer delays of each OM. This is described in the next section.

\section{Transfer delay identification}

The hydraulic systems consist of several reaches, i.e. a part between two measurement points (see Figure 5.a), each reach being composed of Open-Channel Reach Section (OCRS), i.e. a part between two gates, or between a measurement point and a gate or between a gate and a measurement point. Thus, the reach dynamics are modelled with a concurrent Hybrid Automaton cHA (see Figure 5.b) composed of several HA defined for each OCRS. This representation is directly inspired from the concurrent hybrid automata proposed in $[5,6]$. In the study case, the cHA is deterministic. The input of the first automaton $H A_{o_{i}}$ of the first OCRS following $M_{i}$ is the discharge measured on $M_{i}$. The input of the second automaton, $H A_{o_{i}+1}$ is the discharge downstream $H A_{o_{i}}$ minus $q_{n_{i}}$, the discharge setpoint assigned to the gate between this two OCRS. 


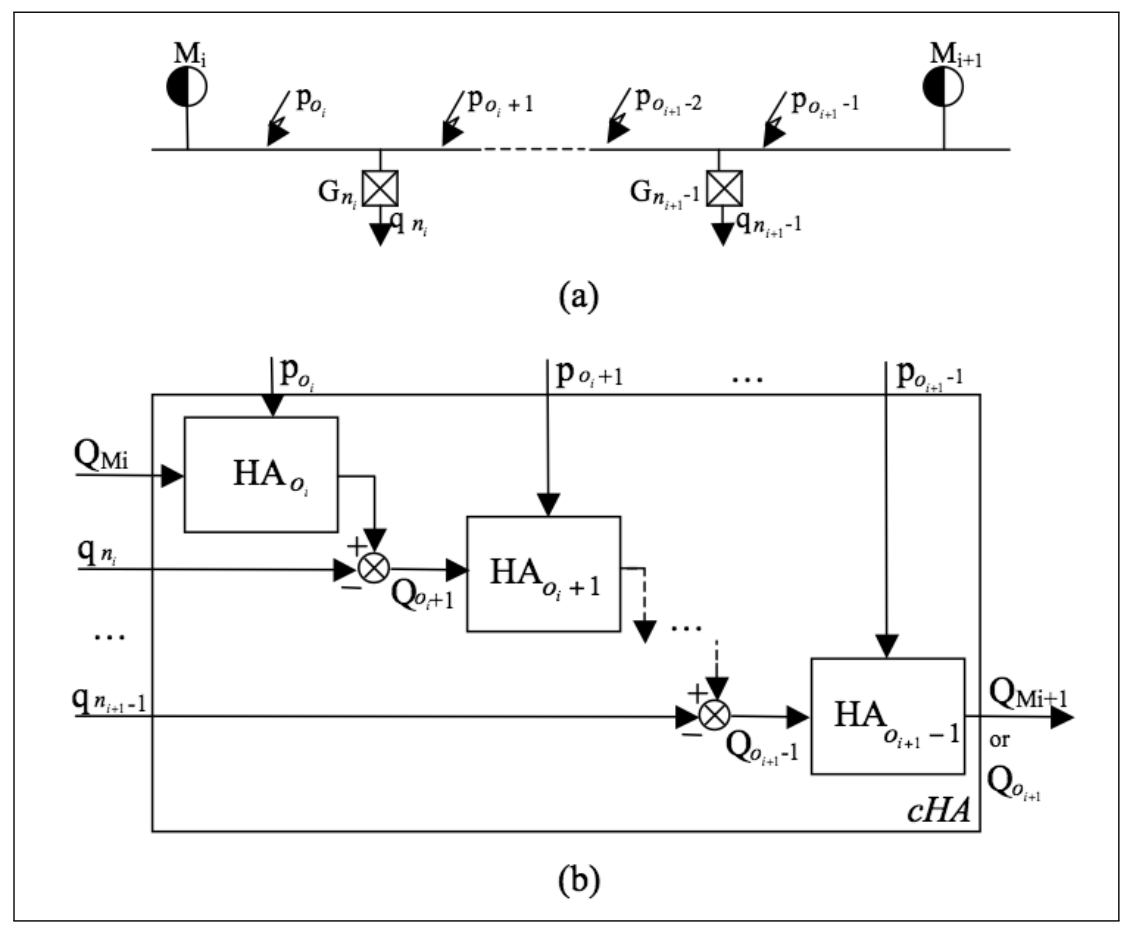

Figure 5: (a) A canal reach and (b) its modelling by concurrent hybrid automaton (cHA).

The OCRS dynamics (see Figure 6.a) are modelled using the multimodelling method described in the previous section, and are represented by a HA (see Figure 6.b). Each state corresponds to one OM identified as transfer function $F_{l}$ given by the relation (6). The transition conditions are defined according to the upstream discharge $Q_{o}$ and the $l_{t h}$ OM boundaries $Q_{i n f_{l}}$ and $Q_{s u p_{l}}$.

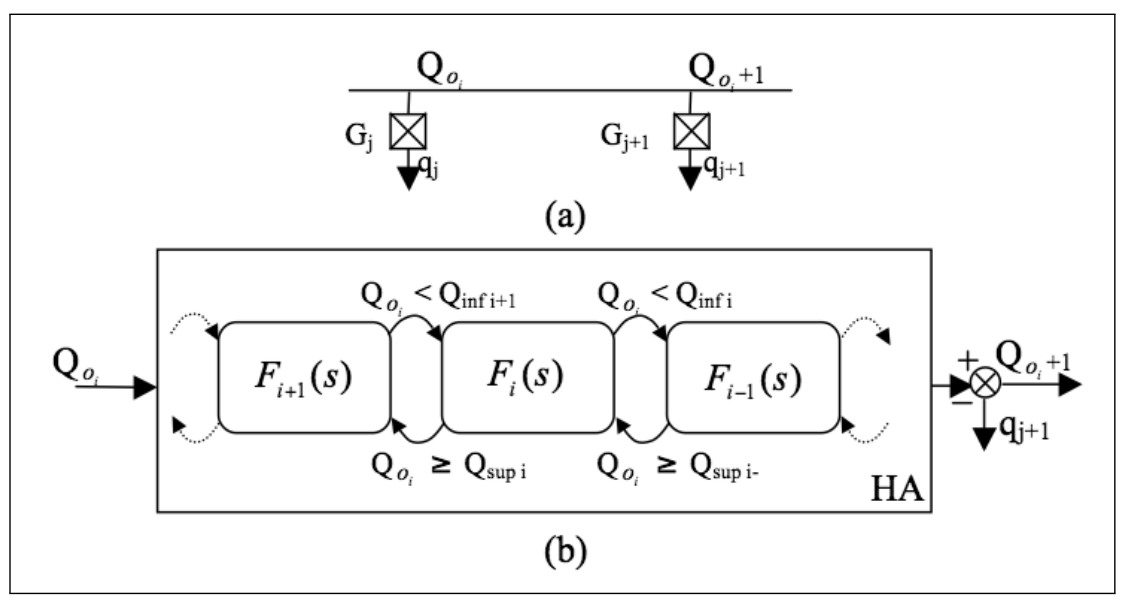

Figure 6: $(a)$ OCRS with index o and $(b)$ its modelling by Hybrid Automaton (HA).

For each OM, the transfer delay $t_{r}$ is obtained from the step response of the corresponding transfer function. It is chosen as the time value for which $\Pi_{Q}$ percent of step is reached. The percentage $\Pi_{Q}$ can be tuned from simulation. In the next section, the HCA strategy is used to valorize water quantities of a hydraulic system subjected to large operating conditions. 
Table 2: Geometrical characteristics of each OCRS.

\begin{tabular}{|c|c|c|c|c|c|c|}
\hline OCRS & $B[\mathrm{~m}]$ & $f$ & $X[\mathrm{~m}]$ & $J[\mathrm{rad}]$ & $Q_{\min }\left[\mathrm{m}^{3} / \mathrm{s}\right]$ & $Q_{\max }\left[\mathrm{m}^{3} / \mathrm{s}\right]$ \\
\hline 1 & 5.73 & 0.79 & 204 & $7.10^{-4}$ & 0.8 & 10 \\
\hline 2 & 5.09 & 0.96 & 702 & $7.10^{-4}$ & 0.8 & 9 \\
\hline 3 & 5.21 & 0.95 & 562 & $6.10^{-4}$ & 0.7 & 7 \\
\hline 4 & 3.72 & 0.94 & 1360 & $5.10^{-4}$ & 0.6 & 5 \\
\hline
\end{tabular}

\section{Simulation results}

The effectiveness of the proposed strategy is shown by simulation on the first reach of the Neste canal located in Gascogne, a french southwestern region. It is composed of one measurement point $M_{1}$, three gates $G_{1}$ to $G_{3}$ and one output considered as a non-controlled gate $G_{4}$ (see Figure 7). This canal reach is composed of four OCRS with trapezoidal profile characterized by the bottom width $B$, the average fruit of the banks $f$, the profile length $X$ and the reach slope $I$ (see Figure 8). The geometrical characteristics and operating conditions of each OCRS are detailed in Table 2. The fourth OCRS, between $G_{3}$ and $G_{4}$, is not modelled, because gate $G_{4}$ is not locally controlled. In the case of a trapezoidal profile, celerity and diffusion coefficients $C$ and $D$ are expressed by the following relation:

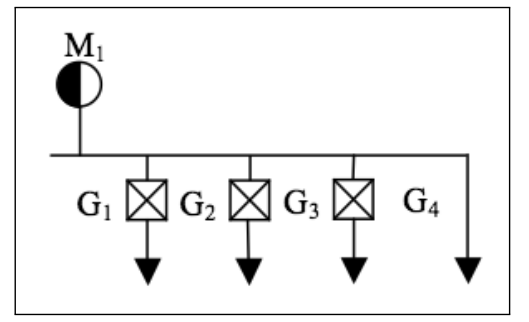

Figure 7: First reach of the Neste canal.

$$
\left\{\begin{array}{l}
C=\frac{Q}{L^{2}}\left[-f+\frac{L}{3}\left(\frac{2 B}{P z}+\frac{5 L}{S}-\frac{2}{z}\right)\right] \\
D=\frac{Q}{2 L J}
\end{array},\right.
$$

where $L=B+2 f z, S=z B+f z^{2}, P=B+2 z \sqrt{1+f^{2}}$.

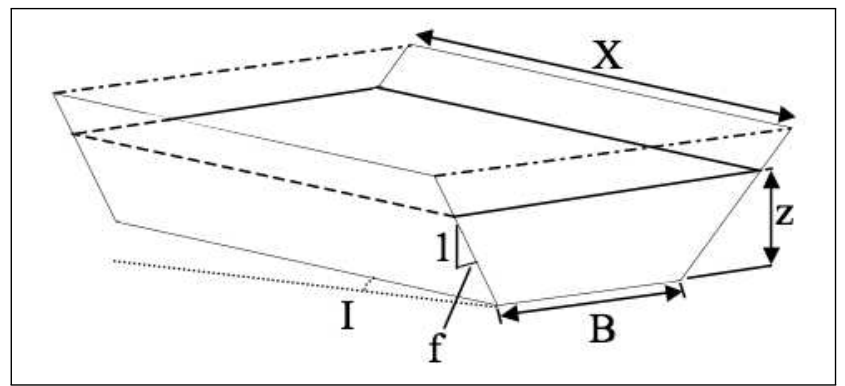

Figure 8: Geometrical characteristics of a trapezoidal profile.

The multimodelling steps which are carried out with $\Pi_{C}=15 \%$ and $\Delta_{C}=0.1$, lead to the determination of three OM for each OCRS. Their corresponding transfer function parameters are given in Table 3 . The transfer delays $t_{r}$ are computed with a value $\Pi_{Q}=63 \%$. This value, chosen by simulation, leads to the best water quantity valorization. 
Table 3: Multiple models of each OCRS.

\begin{tabular}{|c|c|c|c|c|c|c|c|}
\hline OCRS & $Q$ & $Q_{\min }\left[\mathrm{m}^{3} / \mathrm{s}\right]$ & $Q_{\max }\left[\mathrm{m}^{3} / \mathrm{s}\right]$ & $w_{1}$ & $w_{2}$ & $\tau$ & $t_{r}[\mathrm{~s}]$ \\
\hline \hline & 1.6 & 0.8 & 2.4 & 161 & 0 & 0 & 160 \\
\hline 1 & 3.2 & 1.9 & 5.2 & 128 & 0 & 0 & 130 \\
\hline & 7.7 & 4.3 & 10 & 100 & 0 & 0 & 100 \\
\hline \hline & 1.4 & 0.8 & 2.2 & 424 & 24331 & 145 & 575 \\
\hline 2 & 3 & 1.7 & 4.9 & 364 & 0 & 89 & 450 \\
\hline & 7.4 & 4.1 & 9 & 355 & 0 & 0 & 355 \\
\hline \hline & 1.2 & 0.7 & 1.9 & 377 & 0 & 129 & 505 \\
\hline 3 & 2.4 & 1.5 & 4 & 357 & 0 & 29 & 385 \\
\hline & 5.8 & 3.2 & 7 & 320 & 0 & 0 & 320 \\
\hline
\end{tabular}

The Neste canal is supplied with water corresponding to an objective discharge equal to $7 \mathrm{~m}^{3} / \mathrm{s}$. The objective discharges and the weights of each gate are displayed in Table 4. The canal is subjected to strong withdrawals of $3 \mathrm{~m}^{3} / \mathrm{s}$ and $4.5 \mathrm{~m}^{3} / \mathrm{s}$ upstream to $M_{1}$ (see Figure 9.a). This scenario was selected among several simulated scenarii, because it reveals accurately the inversion phenomenon of the discharge tendency. The HCA strategy goals consist in the allocation of the water resource amongst the gates according to their weights, and in the minimization of the discharge discrepancy at gate $G_{4}$. The detection and control periods are selected as $T_{e}=12 \mathrm{~s}$ and $T_{c}=120 \mathrm{~s}$. In Figure 9, the simulation results are shown in continuous line when the transfer delay selection method is used, and in dashed line when the transfer delays are fixed to $100 s$ for the first OCRS, to $355 s$ for the second and to $320 s$ for the third. These transfer delay values are selected according to the operating point of each OCRS, i.e. $7 \mathrm{~m}^{3} / \mathrm{s}$, $5 \mathrm{~m}^{3} / \mathrm{s}$ and $4 \mathrm{~m}^{3} / \mathrm{s}$. They correspond to the third OM of each OCRS.

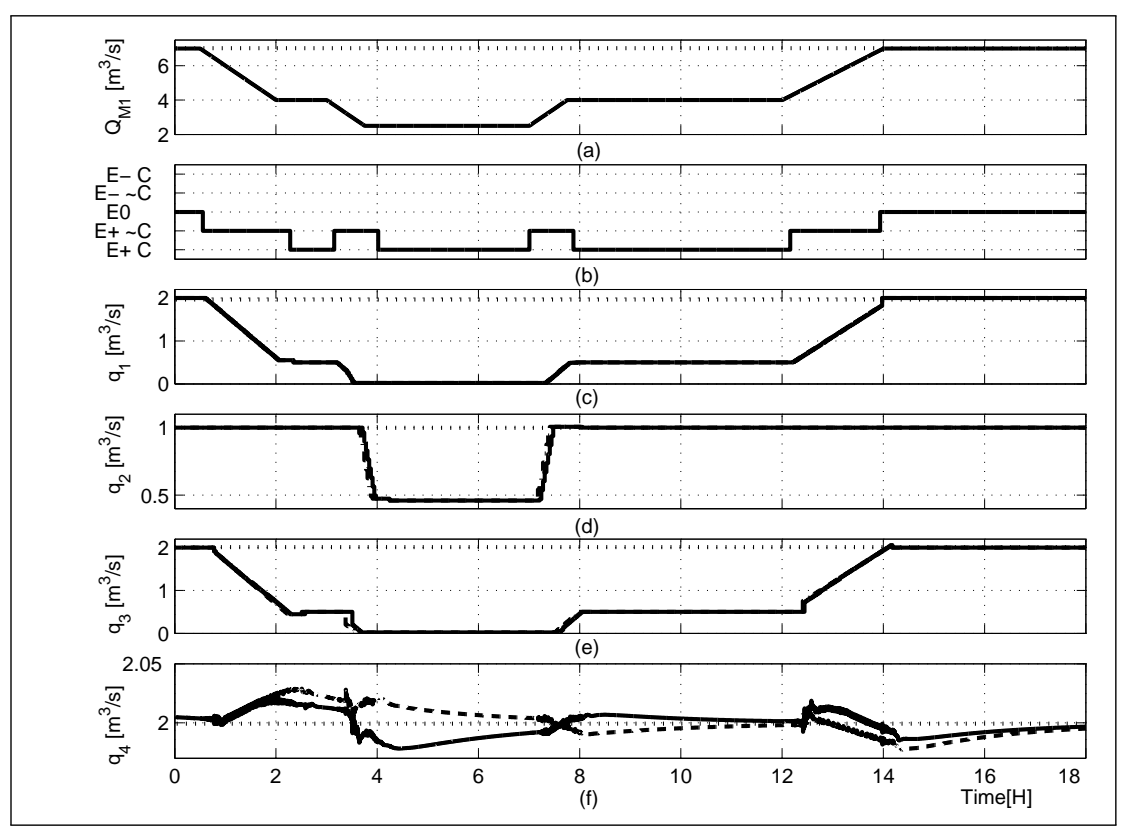

Figure 9: Discharge $Q_{M_{1}}$ (continuous line) and objective discharge $Q_{M_{1} o b j}$ (dotted line) $(a)$, resource states $(b)$, setpoints assigned $q_{j}$ (continuous line when the delay selection method is used, dashed line in other case) and objective discharge $q_{j o b j}$ (dotted line) to $G_{1}(c), G_{2}(d), G_{3}(e)$ and discharge at $G_{4}(f)$. 
Table 4: Gate objective discharges $q_{j o b j}$, gate weights $\lambda_{j}$ and $\mu_{j}$, and minimum and maximum discharges $q_{j_{\min }}$ and $q_{j_{\max }}$.

\begin{tabular}{|c|c|c|c|c|}
\hline Gate & $G_{1}$ & $G_{2}$ & $G_{3}$ & $G_{4}$ \\
\hline$q_{\text {jobj }}\left[\mathrm{m}^{3} / \mathrm{s}\right]$ & 2 & 1 & 2 & 2 \\
\hline$\lambda_{j}$ & 10 & 10 & 4 & - \\
\hline$\mu_{j}$ & 10 & 4 & 10 & - \\
\hline$q_{j_{\min }}\left[\mathrm{m}^{3} / \mathrm{s}\right]$ & 0.02 & 0.1 & 0.02 & 0.15 \\
\hline$q_{j_{\max }}\left[\mathrm{m}^{3} / \mathrm{s}\right]$ & 3.6 & 4.5 & 3.5 & 3 \\
\hline
\end{tabular}

Table 5: Criteria computed when the transfer delay selection method is or not used.

\begin{tabular}{|c|c|c|c|}
\hline Criterion & $\min \left(q_{4}\left[\mathrm{~m}^{3} / \mathrm{s}\right]\right)$ & $\max \left(q_{4}\left[\mathrm{~m}^{3} / \mathrm{s}\right]\right)$ & $V\left[\mathrm{~m}^{3}\right]$ \\
\hline With selection method & 1.9778 & 2.0451 & 541 \\
\hline Without selection method & 1.9763 & 2.0455 & 602 \\
\hline
\end{tabular}

Figure 9 displays the discharges $Q_{M_{1}}$ in (a), the corresponding discharge states in (b), the setpoint assigned at gate $G_{1}$ in (c), $G_{2}$ in (d), $G_{3}$ in (e) and the resulting discharge at $G_{4}$ in (f). The resource in lack measured on $M_{1}$ is allocated on gates $G_{1}$ and $G_{3}$, as long as their setpoints are upper than their respectively minimum discharge characteristics $q_{j_{\min }}$, otherwise it is allocated on gate $G_{2}$ (see Figures 9.c, 9.d and 9.e). Thus, the gates $G_{1}$ and $G_{3}$ are controlled between the $1^{s t}$ and $4^{\text {th }}$ hours, and the gate $G_{2}$ between the $4^{\text {th }}$ and $7^{\text {th }}$ hours.

During all the simulation time, the discharge on $G_{4}$ is close to the objective value of $2 \mathrm{~m}^{3} / \mathrm{s}$ (see Figure 9.f). However, when the transfer delay selection method is not used, the setpoints are always assigned too early. Consequently at $G_{4}$, the discharge is in excess when the water resource decreases on $M_{1}$, and the discharge is in lack when the water resource increases on $M_{1}$. The tendency of $Q_{M_{1}}$ discharge is inverted at $G_{4}$ the end of the canal reach.

The use of the transfer delay selection method improves the performances of the HCA strategy and maintains the tendency. The maximum and minimum discharges reached at $G_{4}$ and the water volume $V$ which was not allocated, are displayed in Table 5. The maximum discharge discrepancy at $G_{4}$ corresponds to $2.26 \%$ of the objective discharge $q_{4 o b j}$ when the transfer delay selection method is used and to $2.28 \%$ in the other case.

\section{Conclusion}

The HCA strategy is adapted to valorize the water resource by allocation and setpoint assignment of open-surface hydraulic system submitted to strong disturbances. A multimodelling method is proposed to identify by a determined number of linear models the open-surface hydraulic systems. Then, a transfer delay selection method is proposed to take into account variable transfer delays by the selection of the right setpoint assignment date according to the system dynamics and to the measured discharge. Finally, the HCA strategy performances are shown by simulation on the first reach of the Neste canal subjected to large operating conditions and to strong disturbances. The study of control stability proof of the HCA strategy is a future goal. 


\section{Bibliography}

[1] Chow, V. T., D. R. Maidment and L. W. Mays. Applied Hydrology. McGraw-Hill. New York, Paris, 1988.

[2] Choy, S. and E. Weyer (2005). Reconfiguration schemes to mitigate faults in automated irrigation channels. CDC-ECC'05, 44 th IEEE Conference on Decision and Control and European Control Conference, Seville, Spain, 12-15 december, pp. 1875-1880, 2005.

[3] Duviella, E., P. Charbonnaud, P. Chiron and F. Carrillo. Supervised internal multi-model control of a dam-gallery open-channel system. CDC-ECC'05, 44 th IEEE Conference on Decision and Control and European Control Conference, Seville, Spain, 12-15 december, pp. 1887-1892, 2005.

[4] Duviella, E., P. Chiron, P. Charbonnaud and P. Hurand. Supervision and hybrid control accommodation for water-asset management. Control Engineering Practice. Vol. 15, Issue 1, pp. 17-27, 2007.

[5] Funiak, S., L. J. Blackmore and B. C. Williams. Gaussian particle filtering for concurrent hybrid models with autonomous transitions. CSAIL Memo, 2006.

[6] Hofbaur, M. W. and B. C. Williams. Hybrid diagnosis with unknown behavioural modes. International Workshop on Principles of Diagnosis (Dx-02), Semmering, Austria, May 2-4, pp. 97-105, 2002.

[7] Litrico, X. and D. Georges. Robust continuous-time and discrete-time flow control of a dam-river system. (i) modelling. Applied Mathematical Modelling, Vol. 23, pp. 809-827,1999.

[8] Litrico, X. and V. Fromion. Design of structured multivariable controllers for irrigation canals. CDCECC'05, 44 th IEEE Conference on Decision and Control and European Control Conference, Seville, Spain, 12-15 december, pp. 1881-1886, 2005.

[9] Malaterre, P.O., D.C. Rogers and J. Schuurmans. Classification of canal control algorithms. Journal of irrigation and drainage engineering, Vol.124, Issue 1, pp. 3-10, 1998.

[10] Puig, V., J. Quevedo, T. Escobet, P. Charbonnaud and E. Duviella. Identification and control of an open-flow canal using LPV models. CDC-ECC'05, 44 th IEEE Conference on Decision and Control and European Control Conference, Seville, Spain, 12-15 december, pp. 1893-1898, 2005.

Eric Duviella

Ecole des Mines de Douai

Département Informatique et Automatique 941, rue Charles Bourseul, BP 10838, 59508 Douai Cedex, France Phone: 33.3.27.71.21.02 ; Fax : 33.3.27.71.29.80

E-mail: duviella@ensm-douai.fr

Pascale Chiron

Laboratoire Génie de Production

Ecole Nationale d'Ingénieurs de Tarbes 47, Avenue d'Azereix, BP1629, 65016 Tarbes Cedex, France Phone: 33.5.62.44.27.69 ; Fax : 33.5.62.44.27.08

E-mail: pascale.chiron@enit.fr Philippe Charbonnaud

Laboratoire Génie de Production Ecole Nationale d'Ingénieurs de Tarbes 47, Avenue d'Azereix, BP1629, 65016 Tarbes Cedex, France 
Phone: 33.5.62.44.27.34; Fax : 33.5.62.44.27.08

E-mail: philippe.charbonnaud@enit.fr

Received: January 29, 2007

Revised: May 22, 2007

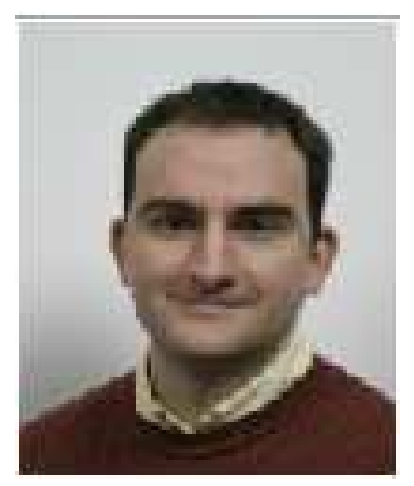

Eric Duviella was born in France in 1978. In 2001, he received the Diploma in engineering from the National Engineering School, Tarbes, France. He received the Ph.D. degree from the Institut Polytechnique, Toulouse, France, in 2005. Since 2007, he has been an Assitant Professor in the Department Informatic and Automatic of the Ecole des Mines, Douai, France. He has published around 15 papers in journals or international conferences. His research interests include modelling, hybrid dynamical systems, supervision, reactive control strategy.

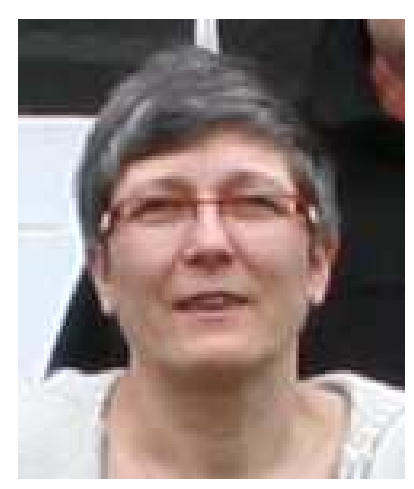

Pascale Chiron was born in France in 1961. She is assistant professor at the Ecole Nationale d'Ingénieurs de Tarbes, France since 2000. She has obtained his PhD in 1989 at Ecole Centrale in Nantes on 'matching and similarity criterion in medical imaging'. Now, she is involved in the theme 'Planning, Control, Supervision and Distributed Simulation' in the 'Automated Production' Group of Laboratoire de Génie de Production. Her domains of interest are modelling and simulation for system control.

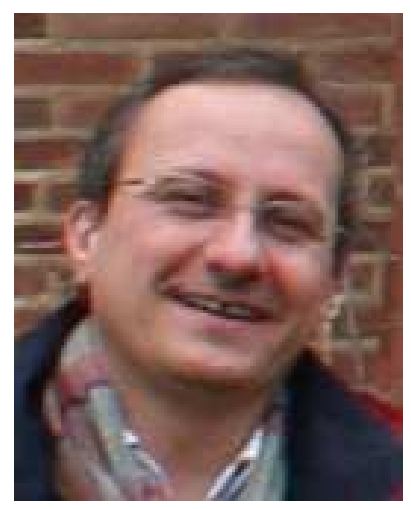

Philippe Charbonnaud was born in France in 1962. Since 2003, he is full Professor at the Ecole Nationale d'Ingénieurs de Tarbes. $\mathrm{He}$ is coordinator of the Spanish-French project GEPREDO dealing with the management of hydrographical networks and the predictive management of the water resource. The main contribution lies in the development of a hybrid control method applied to distributed systems. His main topic of interest concerns the real-time decision support systems, and more particularly supervision and control accommodation of distributed systems. 